\title{
What is Critical Diagnostic Role of Adenoid Hypertrophy and Adult-Onset Otitis Media with Effusion in Clinically Asymptomatic Nasopharyngeal Carcinoma? \\ OI Ozdamar, GO Acar, M Tekin
}

\begin{abstract}
Objectives: The aim of this study is to verify the critical role of adenoid hypertrophy and otitis media with effusion in adult patients in relation to diagnostic importance for patients with clinically asymptomatic nasopharyngeal carcinoma.

Methods: One hundred and six adult patients met our criteria out of 256 cases underwent nasopharyngeal biopsy for the suspect of nasopharyngeal malignancy in our clinic between January 2009 and July 2014 were enrolled in this retrospective study. We divided the patients into two groups according to patients with or without synchronous presence of otitis media with effusion in addition to adenoid hypertrophy.
\end{abstract}

Results: Two patients out of 68 (2.9\%) in the first (only adenoid hypertrophy) group and in one patient out of $38(2.6 \%)$ in the second group had nasopharyngeal carcinoma. There was no statistically significant difference.

Conclusion: We found that asymmetric adenoid hypertrophy in adult patients seems an important risk factor, and we strongly suggest that it needs biopsy for the suspect of nasopharyngeal carcinoma whether they synchronously have or not otitis media with effusion even though in the absence of other clinical symptoms to arouse suspicion about a nasopharyngeal malignancy such as neck mass, epistaxis, cranial nerve impairment.

Keywords: Adult, adenoid hypertrophy, nasopharyngeal carcinoma, otitis media with effusion

From: Department of Otorhinolaryngology- Head and Neck Surgery, Goztepe Training and Research Hospital, Istanbul Medeniyet University, Istanbul, Turkey.

Correspondence: Dr O Ozdamar, Department of Otorhinolaryngology- Head and Neck Surgery, Goztepe Training and Research Hospital, Istanbul Medeniyet University, Erkin cd. No: 1 Goztepe, 34730 / İstanbul, Turkey. Fax:+90216 4674951, e-mail: osmanilkay73@yahoo.com

*This study was presented as a poster presentation in 29. Politzer Society Meeting, 1317/11/2013, Antalya, Turkey 


\section{INTRODUCTION}

Nasopharyngeal carcinoma (NPC), arises in the epithelium of the nasopharynx, is a rare malignant tumor in the United States and Europe that is only 1 per 100,000 population with a male predominance by a margin of about 2 to 1 , but the incidence is approximately 30 times higher in Taiwan, Hong Kong, and southern China (especially Guangdong province) where is accepted as endemic region (1).

Although adult onset-otitis media with effusion (AO-OME) is a disease that is admitted to be unique to childhood, it can be seen in any age group. Possible causes of AOOME are adenoid hypertrophy $(\mathrm{AH})$, nasal problems, acute respiratory tract infection, acute otitis media, secondary smoking $(2,3)$. Rare diagnosis of AO-OME in adults compared to children, which may be an early symptom of a probable nasopharyngeal carcinoma (NPC), warrant further evaluation for underlying a malignant disease; especially, if it is on one ear.

NPC can cause OME through the following proposed pathologic mechanisms: 1- obstruction of the Eustachian tube directly with a mass effect of the tumor, 2- tumor invasion of tensor veli palatine muscle that results in obstruction or dysfunction of Eustachian tube (4). So, flexible endoscopic nasopharyngeal examination of adult patients with AO-OME require to rule out of a malignancy.

In this study, our aim is to verify the critical role of adenoid hypertrophy and otitis media with effusion in adult patients in relation to diagnostic importance for patients with clinically asymptomatic nasopharyngeal carcinoma in a non-endemic geographic area for the disease by comparing biopsy results of two study groups: group 1- the patients only had symmetrical or asymmetrical adenoid hypertrophy (AH), and group 2- the patients synchronously had symmetrical or asymmetrical AH and otitis media with effusion (OME). Thus, unessential nasopharyngeal biopsies that would have been taken in suspected patients could be decreased in relation with clinically asymptomatic NPC. 


\section{SUBJECTSS AND METHODS}

The retrospective research protocol was approved by local Clinical Research Ethics Committee. One hundred and six patients, who underwent a nasopharyngeal biopsy with/without insertion of grommet ventilation tube to one or both ears between January 2000 and July 2014 at a tertiary health care center, were analyzed by this retrospective study.

All of the patients were older than 18 years old, ranging from 18 to 76 year-old. They were confirmed to have $\mathrm{AH}$ by magnetic resonance imaging (MRI) and flexible nasopharyngoscopy but a suspected lesion was not detected in any patient. Patients without MRI were excluded from the study to assure that the patients were not included having submucosal tumor undetected with imagination methods, thus only patients were included clinically asymptomatic NPC by means of physical examination and imaging methods. Otomicroscopic examination, tympanometry and pure tone audiometry were performed for each patient who had OME. All of the patients had undergone nasopharyngeal biopsy to exclude NPC. We divided the patients into two groups according to synchronous presence or absence of OME in addition to AH. In the first group; there were 68 patients, who only had $\mathrm{AH}$. In the second group, there were 38 patients, who had OME in addition to AH. Patients with OME were performed myringotomy and insertion of grommet ventilation tube as a standard treatment (Table 1).

\section{Patients with AH}

In this group of adult patients had solely $\mathrm{AH}$. $\mathrm{AH}$ was either symmetrical and generalized, or asymmetrical in which only one side of nasopharynx was hypertrophied. All of the patients underwent nasopharyngeal biopsy under general anesthesia. Malignancies other than nasopharyngeal carcinoma were excluded because of our focusing on only NPC.

Thirty one patients were male, and 37 patients were female. The mean age of the patients was 43.2 year-old, with a range of 18 to 78 . The cases who had signs and symptoms 
related with a high suspicion for a malignancy, such as epistaxis, pathologic lymphadenopathy in the neck, malignant lesion detected at the nasopharyngeal area with flexible fiberoptic examination and/or with magnetic resonance imaging (MRI) were excluded from the study. Only patients with apparently normal except than asymmetrical or generalized symmetrical non-specific AH were included.

\section{Patients with AH and OME}

In this group of adult patients had OME in addition to $\mathrm{AH}$; $\mathrm{AH}$ was either symmetrical and generalized, or asymmetrical in which only one side of nasopharynx was hypertrophied.

Twenty-two patients were male, and 16 patients were female. The mean age of the patients was 46.6 year-old, with a range of 18 to 72 year-old. As in the first group, the cases with high suspected signs and symptoms were excluded from the study. Only patients with apparently normal except non-specific AH with synchronous OME were included.

\section{Statistical analysis}

Data were analyzed using a commercially available statistics software package (SPSS) for Windows 15.0. The Pearson's chi-squared and Fisher's exact tests were used to analyze discrete variables to compare the two groups; in all analyzes, values of $\mathrm{p}<0.05$ were considered statistically significant.

\section{RESULTS}

Two patients out of 68 revealed a NPC in the first group (both of them were nonkeratinizing squamous cell carcinoma, WHO type II). The carcinoma ratio was 2/68 (2.9 $\%)$. In the second group, one patient out of $38(2.6 \%)$ was detected as NPC (nonkeratinizing squamous cell carcinoma, WHO type II). There was no statistically significant difference 
between the groups $(\mathrm{p}>0.05)$, (Table2). Overall 3 out of $106(2.8 \%)$ patients underwent nasopharyngeal biopsy had diagnosed as nasopharyngeal carcinoma.

In group 1; Fifty five patients had symmetrical generalized $\mathrm{AH}$, and remaining 15 patients had asymmetrical AH in whom seven were in the right, and 6 were in the left side. Nearly half of the patients $(33 / 68 ; 48.5 \%)$ had no symptom, and AH in these subjects were detected incidentally on MRI that were performed by neurology and neurosurgery clinics for other purposes, mainly differential diagnosis of chronic headache. These cases were consulted to our clinic, and all patients underwent flexible endoscopic examination. Other 35 patients $(51.5 \%)$ applied to our clinic for nasal obstruction and postnasal dripping, and $\mathrm{AH}$ detected with endoscopic examination of the nasopharynx but a suspected lesion was not established in any patient. MRI findings were also seemingly normal except than $\mathrm{AH}$ in all patients.

Two out of $68(2.9 \%)$ cases were revealed to have carcinoma of the nasopharynx with biopsy results. Both of them had asymmetric $\mathrm{AH}$ in whom were right-sided with unremarkable medical history in one female patient and left-sided in the other male patient. Female patient was consulted to our clinic for $\mathrm{AH}$ as an incidental finding on MRI. On the other hand, male patient applied to our clinic for nasal obstruction, which was progressed for the last 6 months. The patient had undergone a septoplasty for septal deviation in the same surgical session. None of the patients' pathologic results who had symmetric generalized AH in the nasopharynx were NPC (Table 3).

In group 2; Twenty five patients $(25 / 38 ; 65.8 \%)$ had symmetrical, generalized $\mathrm{AH}$, and remaining 13 patients $(13 / 38 ; 34.2 \%)$ had asymmetrical $\mathrm{AH}$ in which seven were in the right, and 6 were in the left side. All of the patients had applied to our clinic for the complaints of hearing loss, aural fullness, and tinnitus with/without nasal obstruction. AH detected with endoscopic examination of the nasopharynx but a suspected lesion was not 
established in any patient. MRI findings were also seemingly normal except than non-specific $\mathrm{AH}$ with synchronously present $\mathrm{OME}$ in all patients. One out of $38(2.6 \%)$ cases was revealed to have carcinoma of the nasopharynx with biopsy results. The patient was a male patient who had a left-sided asymmetric AH. The patient applied to our clinic for nasal obstruction, which was progressed for the last 6 months, and a left-sided asymmetric AH was detected through an endoscopic examination of the nasopharynx. None of the patients' pathologic results who had symmetric generalized AH in the nasopharynx were NPC (Table $3)$.

\section{DISCUSSION}

Nasopharyngeal biopsy performed in otorhinolaryngology practice under general or local anesthesia is not an uncommon surgical intervention in patients with suspected malignant disease of the nasopharynx. Nevertheless, the high rates of negative biopsy results are questioned validity of its implementation, which are waste of time and cost in these circumstances. We found that asymmetric adenoid hypertrophy in adult patients seems an important risk factor, and we strongly suggest that it needs biopsy for the suspect of nasopharyngeal carcinoma whether they synchronously have or not otitis media with effusion even though in the absence of other clinical symptoms to suspect a nasopharyngeal malignancy.

Two well-documented important clinical features of NPC are firstly; high incidence in some geographic areas, such as China, Southeast Asia and North Africa which have an incidence of up to thirty-folds than low-incidence geographical areas including United States and Europe, and secondly; high incidence in some races in whom live in high-occurrence geographic areas (5). In these patients, nasopharyngeal biopsy could be encountered to rule 
out of a malignancy in clinically suspected situations, because early detection of a NPC would improve patient's prognosis, and quality of life.

Hsieh CC et al. (6) had evaluated a large number of patients who underwent nasopharyngeal biopsy for the suspect of nasopharyngeal carcinoma, and they assessed various clinical symptoms and signs (such as; nasopharyngeal mass, neck mass, epistaxis, OME, headache, cranial nerve involvement) with the biopsy results by dividing the patients to cancerous and noncancerous groups. They detected that statistically significant differences were found only for the nasopharyngeal mass, neck mass and age between the groups, and the only significant variable in cancerous group was neck mass. They found that nasopharyngeal mass had the highest sensitivity (90.7\%), but the lowest specificity $(28.4 \%)$ due to high rate of misdiagnosis of nasopharyngeal lymphoid hyperplasia as NPC when examined with conventional white-light endoscopes but they had not discriminated the symmetric and asymmetric $\mathrm{AH}$ in cancerous and noncancerous groups. On the other hand, an important clinical feature of this malignant disease is possibility of a submucosal tumor overlying normal looking mucosa which needs taking a biopsy including submucosal deep tissue up to a few millimeters (7).

A novel optical technique to visualize nasopharynx, Narrow-band imaging (NBI), was also introduced in addition to conventional white-light endoscopes (8, 9). NBI and conventional endoscopes were compared for differentiation rates of AH and NPC in 79 consecutive adult patients (9). They concluded that NBI was superior to white-light endoscope for the detection of benign AH but not that of NPC. However, NBI was not performed in our cases when we evaluated patients' files and electronic charts.ME of childhood is mostly bilateral, and have numerous reasons except than tumors but it may be an early sign of a NPC in adults, which is nearly almost unilateral occurrence. This warrants performing endoscopic examination of the nasopharynx to exclude a nasopharyngeal 
malignancy. However, Gaze et al. (10) detected nasopharyngeal neoplasm in adults with only symptom of OME as an incidence of 1.4\%; similarly, they found in the second group of adult patients who had nasopharyngeal tumor presenting with only complaint of OME as an incidence of $1.5 \%$. It was concluded that the expected rate to disclose a diagnosis of NPC with isolated OME in adult patients has been presented in the literature to be between $0.4 \%$ and $5.7 \%$ depending on studied population $(4,10-12)$. They suggested using clinical judgment with other clinical findings whether an endoscopic nasopharyngeal examination is necessary or not in these patients.

Deeb and Ashktorab (13) concluded that bilateral adult-onset OME cases were related with benign clinical conditions, but unilateral cases needed nasopharyngoscopy for a suspect of a nasopharyngeal malignancy. Glynn et al. (14) presented 85 adult patients with isolated serous otitis media who underwent nasopharyngeal biopsy, and serous otitis media were unilateral in $59(69 \%)$ patients and bilateral in $26(31 \%)$ patients. They detected that 3 out of 59 unilateral OME cases were NPC but 1 out of 26 bilateral serous otitis media case was lymphoma. We agree that adult patients with unilateral OME need endoscopic examination of the nasopharynx to rule out a malignancy. . Additionally, OME due to NPC is not improved with medical treatment or spontaneously that needs endoscopic examination of the nasopharynx. For this reason, we excluded the patients in whom OME was improved with medical treatment or spontaneously that did not need ventilation tube insertion.

All of the mentioned studies were performed in low-incidence geographic areas. Our results in AH without/with OME are higher for both of our study groups which are 2.9\% and $2.6 \%$ respectively. Possible cause may be AH that is present in all our patients. Moreover, only $\mathrm{AH}$ group was slightly higher than the $\mathrm{AH}$ and $\mathrm{OME}$ group; $2.9 \%$ versus $2.6 \%$ but there was no clinically significant difference ( $\mathrm{p}>0.05)$. Ho et al. (4) detected that 5 patients out of 87 were diagnosed as NPC with an incidence of $5.7 \%(5 / 87)$. Nevertheless, they did not 
mention about the $\mathrm{AH}$ that was detected by endoscopic nasopharyngeal examination and/or MR imagination technique. Additionally, they concluded that the high percentage of NPC was the result of endemicity for the disease in their population.

MRI ot the nasopharynx is not only important for detection of a malignancy but also for staging of NPC. Cui et al. (15) found that MRI is superior to clinical detection of trigeminal nerve involvement in untreated NPC patients. Tumor might invade deep anatomic structures such as skull base, trigeminal nerve, cavernous sinus, intracranial region by extending superiorly and laterally (14). Therefore, MRI is necessary in suspected patients with a normal looking nasopharyngeal mucosa with or without adenoid hypertrophy.

\section{CONCLUSION}

We detected that asymmetric adenoid hypertrophy in adult patients seems an important risk factor needing biopsy for the suspect of NPC whether it is present or not synchronously with OME. We conclude the followings related with clinically asymptomatic NPC in a lowincidence geographic area for the disease;

1- Asymmetric $\mathrm{AH}$ in adult patients has a high risk for NPC, and we strongly suggest that this finding needs biopsy to detect a malignancy earlier if it is present.

2- NBI endoscopic examination of the nasopharynx coupled with conventional whitelight endoscopic examination has a higher chance to detected benign adenoid lymphoid hypertrophy; therefore, it might decrease the unnecessary nasopharyngeal biopsies.

3- Generalized symmetric $\mathrm{AH}$ as with OME in adult patients has relatively low risk for NPC, and need of biopsy of the nasopharynx in the absence of other signs and symptoms for the suspect of a malignancy; such as neck mass, a tumorous lesion detected by the endoscopic examination of the nasopharynx, seems that other factors are important including clinical 
justification and experience of the surgeon and also patient related factors such as age, sex, medical history etc. AO-OME in non-endemic geographic regions and in low-race populations for the malignancy could be due to benign diseases, such as allergic conditions and chronic infections. 


\section{REFERENCES}

1. Jeyakumar A, Brickman TM, Jeyakumar A, et al. Review of nasopharyngeal carcinoma. ENT-Ear, Nose Throat J 2006; 85: 168-84.

2. Kraemer MJ, Richardson MA, Weiss NS, et al. Risk factors for persistent middle ear effusions. JAMA 1983; 249: 1022-5.

3. Chao WY, Wang CF, Chang SJ. Ventilation tube in adults with middle-ear effusion. J Otolaryngol 1999; 28: 278-81.

4. Ho KY, Lee KW, Chai CY, et al. Early recognition of nasopharyngeal cancer in adults with only otitis media with effusion. J Otolaryngol Head Neck Surg 2008; 37: 362-5.

5. Chang ET, Adami HO. The enigmatic epidemiology of nasopharyngeal carcinoma. Cancer Epidemiol Biomarkers Prev 2006; 15: 1765-77.

6. Hsieh CC, Wang WH, Lin YC, et al. A large-scale study of the association between biopsy results and clinical manifestations in patients with suspicion of nasopharyngeal carcinoma. Laryngoscope 2012; 122: 1988-93.

7. Waldron J, Van Hasselt CA, Wong KY. Sensitivity of biopsy using local anesthesia in detecting nasopharyngeal carcinoma. Head Neck 1992; 14: 24-7.

8. Lin YC, Wang WH. Narrow-band imaging for detecting early recurrent nasopharyngeal carcinoma. Head Neck 2011; 33: 591-4.

9. Wang WH, Lin YC, Weng HH, et al. Narrow-band imaging for diagnosing adenoid hypertrophy in adults: a simplified grading and histologic correlation. Laryngoscope. 2011; 121: 965-70.

11. Gaze MN, Keay DG, Smith IM, et al. Routine nasopharyngeal biopsy in adult secretory otitis media. Clin Otolaryngol Allied Sci 1992; 17: 183-4.

12. Dang PT, Gubbels SP. Is nasopharyngoscopy necessary in adult-onset otitis media with effusion? Laryngoscope $2013 ; \mathbf{1 2 3}$ : 2081-2. 
14. Dempster JH, Simpson DC. Nasopharyngeal neoplasms and their association with adult onset otitis media with effusion. Clin Otolaryngol Allied Sci 1988; 13: 363-5.

15. Deeb ZE, Ashktorab S. In reference to "is nasopharyngoscopy necessary in adultonset otitis media with effusion?". Laryngoscope 2014; 124: E445.

16. Glynn F, Keogh IJ, Ali TA, Timon CI, Donnelly M. Routine nasopharyngeal biopsy in adults presenting with isolated serous otitis media: is it justified? J Laryngol Otol 2006; 120: 439-41.

17. Cui C, Liu L, Ma J, Liang S, Tian L, Tang L, Li L. Trigeminal nerve palsy in $n$ asopharyngeal carcinoma: correlation between clinical findings and magnetic resonance imaging. Head Neck 2009; 31: 822-8. 
Table 1: Patients distribution of $\mathrm{AH}$ with and without $\mathrm{OME}$, and side of ventilation tube insertion are shown

\begin{tabular}{|c|c|c|}
\hline & Patients Number & VT Side \\
\hline Group 1 (AH w/o OME) & 68 & None \\
\hline Group 2 (AH w OME) & 38 & \\
\hline Right & & 11 \\
\hline Left & & 14 \\
\hline Bilateral & & 13 \\
\hline Total & 106 & 38 \\
\hline
\end{tabular}

AH: Adenoid Hypertrophy, OME: Otitis media with effusion, VT: Ventilation tube

Table 2: Comparison of patients with only $\mathrm{AH}$ (group 1) and patients with $\mathrm{AH}$ and $\mathrm{OME}$ (group 2)

\begin{tabular}{|c|c|c|c|c|c|}
\hline & Value & df & $\begin{array}{l}\text { Asymp. Sig. } \\
\text { (2-sided) }\end{array}$ & $\begin{array}{c}\text { Exact Sig. } \\
\text { (2-sided) }\end{array}$ & $\begin{array}{c}\text { Exact Sig. } \\
\text { (1-sided) }\end{array}$ \\
\hline Pearson Chi-Square & ,008 & 1 & ,927 & & \\
\hline Continuity Correction &, 000 & 1 & 1,000 & & \\
\hline Likelihood Ratio & ,009 & 1 & ,926 & & \\
\hline Fisher's Exact Test & & & & 1,000 & ,708 \\
\hline Linear-by-Linear Association &, 008 & 1 & ,927 & & \\
\hline $\mathrm{N}$ of Valid Cases & 106 & & & & \\
\hline
\end{tabular}


Table 3: Distribution of patients in relation with side of AH and biopsy results are shown.

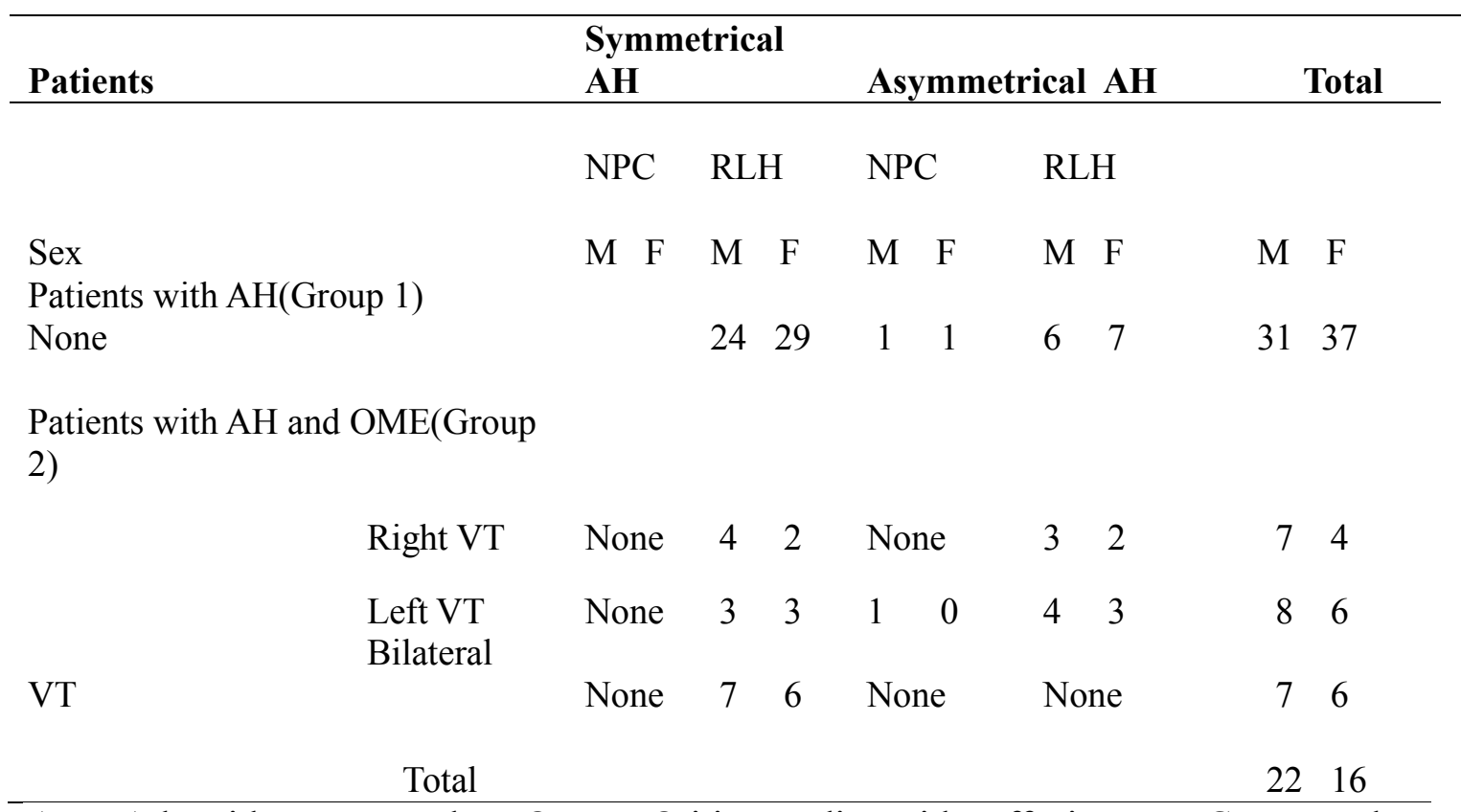

AH: Adenoid Hypertrophy, OME: Otitis media with effusion, NPC: Nasopharyngeal carcinoma, RLH: Reactive lymphoid hyperplasia, VT: Ventilation tube, M: Male, F: Female. 